TTP96-26円

hep-ph/9607259

July 1996

\title{
Higher-Order Hadronic Contributions to the Anomalous Magnetic Moment of Leptons
}

\author{
Bernd Krause \\ Institut für Theoretische Teilchenphysik, \\ Universität Karlsruhe, D-76128 Karlsruhe, Germany
}

\begin{abstract}
Higher-order hadronic contributions to the anomalous magnetic moment of the electron, muon, and $\tau$ lepton are considered in detail. As a main result we find a reduction by $-11 \times 10^{-11}$ for the g- 2 of the muon as compared to previous calculations. Analytical expressions for the kernel functions of higher-order hadronic effects are presented. We employ the method of asymptotic expansions to calculate kernel functions analytically in terms of a series expansion in the lepton mass.
\end{abstract}

\footnotetext{
${ }^{1}$ The complete postscript file of this preprint, including figures, is available via anonymous ftp at ttpux2.physik.uni-karlsruhe.de (129.13.102.139) as /ttp96-26/ttp96-26.ps or via www at http://ttpux2.physik.uni-karlsruhe.de/cgi-bin/preprints/ Report-no: TTP96-26
} 
The investigation of the anomalous magnetic moment of the muon has received considerable interest recently due to the upcoming BNL experiment [1]]. The main goal of this experiment is the confirmation of electroweak loop effects as predicted by the Standard Model. At the same time, deviations may be an indication of physics beyond the Standard Model. It is therefore necessary to fix the Standard Model prediction as precisely as possible.

In this context we note that not only electroweak loop effects but also higher-order hadronic contributions come into the range of experimental detection. The designated experimental uncertainty of $\pm 40 \cdot 10^{-11}$ in the Brookhaven experiment allows for an establishment of higher-order hadronic effects by at least 4 standard deviations. For the electron the situation is similar: from improvements of the Penning-trap technique, experimentalists expect a reduction of the present experimental uncertainty from $\Delta a_{e}= \pm 4.0 \cdot 10^{-12}$ to $\Delta a_{e}= \pm 0.6 \cdot 10^{-12}$ [2], in which case hadronic contributions to $a_{e}=\left(g_{e}-2\right) / 2$ would be a $2 \sigma$ effect.

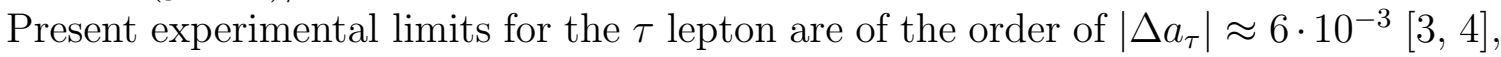
nearly three orders of magnitude below the expected hadronic effects.

The purpose of the present work is to calculate higher-order hadronic diagrams for the electron, muon, and $\tau$ lepton. A new method for the computation of kernel functions is presented. Combined with previous work we now provide a complete set of analytic kernel functions that allow a numerically reliable evaluation of higher-order hadronic contributions to the g-2 of leptons.

This work is based on a recent analysis of experimental data by Eidelman and Jegerlehner [5]. We follow this reference in the treatment of integration over hadronic data. In particular, we also use the trapezoidal rule for the integration over the $\rho$ resonance. Higher resonances are included using the narrow width approximation. The set of kernel functions presented here can, however, also be used for other parametrizations of hadronic data.

The relevant Feynman diagrams are depicted in fig. (2). As in the case of the leading order hadronic correction (fig. 1), these diagrams cannot be computed in QCD perturbation theory. Instead, experimental data on the branching ratio $R(s)$ for $e^{+} e^{-} \rightarrow$ hadrons can be used to compute the diagrams of figs. (1) and $(2 \mathrm{a}-\mathrm{c})$. This is not possible for the so-called light-by-light-diagram (fig. 3). The latter has been computed recently for the muon by two groups using the extended Nambu-JonaLasinio model [6, 7]. It will not be discussed in the present paper.

The paper is organized as follows: In the first section we discuss the calculation of kernel functions and collect relevant formulae. In the following sections we apply these results to the computation of higher-order hadronic contributions to the muon, electron, and tau, respectively. Whenever possible, different methods (analytical and numerical) have been used as a cross-check. 


\section{Kernel Functions}

The common feature of all hadronic contributions discussed in this paper will be that they are self-energy insertions in the photon propagator. We introduce the polarization function $\Pi_{\mu \nu}$ for the hadronic insertion with

$$
\begin{aligned}
\Pi_{\mu \nu}(q) & =i\left(q_{\mu} q_{\nu}-g_{\mu \nu} q^{2}\right) \Pi\left(q^{2}\right) \\
\Pi\left(q^{2}\right) & =-\frac{q^{2}}{\pi} \int_{4 m_{\pi}^{2}}^{\infty} \frac{d s}{s} \frac{\operatorname{Im} \Pi(s)}{q^{2}-s} .
\end{aligned}
$$

The substitution rule from a photon propagator to a renormalized propagator with hadronic insertion follows:

$$
-i \frac{g_{\mu \nu}}{q^{2}} \rightarrow \frac{1}{\pi} \int_{4 m_{\pi}^{2}}^{\infty} d s \frac{\operatorname{Im} \Pi(s)}{s}\left(-i \frac{g_{\mu \nu}}{q^{2}-s}\right) .
$$

The calculation of higher-order kernel functions reduces to the computation of two-loop QED diagrams where one photon is massless and the other photon has the effective mass $\sqrt{s}$. The $s$-integration, which has been shifted to the very end, is performed over experimental data using

$$
R(s)=\frac{\sigma\left(e^{+} e^{-} \rightarrow \text { hadrons }\right)}{\sigma_{0}\left(e^{+} e^{-} \rightarrow \mu^{+} \mu^{-}\right)}=12 \pi \operatorname{Im\Pi }(s)
$$

and the Born cross section $\sigma_{0}\left(e^{+} e^{-} \rightarrow \mu^{+} \mu^{-}\right)=\frac{4 \pi \alpha^{2}}{3 s}$. The leading-order hadronic contribution (fig. 1) to the anomalous magnetic moment of a lepton $a_{l}$ is given by

$$
a_{l}^{(1)}=\frac{1}{3}\left(\frac{\alpha}{\pi}\right)^{2} \int_{4 m_{\pi}^{2}}^{\infty} d s \frac{R(s) K^{(1)}(s)}{s}
$$

with

$$
K^{(1)}(s)=\int_{0}^{1} d x \frac{x^{2}(1-x)}{x^{2}+(1-x) \frac{s}{m^{2}}},
$$

where $m$ denotes the mass of the external fermion; explicit expressions for $K^{(1)}(s)$ can be found e.g. in [5, 8]. After renormalization, the higher-order kernel functions for the diagrams in fig. $(2 \mathrm{a}, \mathrm{b})$ can be defined in a similar way:

$$
a_{l}^{(2)}=\frac{1}{3}\left(\frac{\alpha}{\pi}\right)^{3} \int_{4 m_{\pi}^{2}}^{\infty} d s \frac{R(s) K^{(2)}(s)}{s} .
$$

Once the functions $K^{(2)}(s)$ are known analytically, the higher-order hadronic contributions to the g-2 of leptons can be computed on an equal footing with the leading order (e.g. with the same systematic error).

The present situation concerning $K^{(2)}$ is the following: For the class of diagrams in fig. (2a), a complete analytical result for the kernel function has been given in 9] (eq.(3.21)). In that reference an expansion to first order in $\mathrm{m}^{2} / \mathrm{s}$ has also been 
provided. This expansion has been employed for the calculation of hadronic contributions from diagrams (2a). In the case of diagrams (2b) and (2c) the integral representations eq.(9), 13) have been used.

As a new approach we employ the method of asymptotic expansions [10]. This technique has recently been applied to the calculation of two-loop electroweak corrections to the g-2 of the muon [11, 12]. The calculation of hadronic kernel functions for the muon and the electron can be treated in exactly the same way.

The result is an expansion in the small parameter $\mathrm{m}^{2} / \mathrm{s}$, i.e. the ratio of the muon (or electron) mass $\mathrm{m}$ and the energy $\sqrt{s}$ of the final dispersion integral over hadronic data. This final integration is numerically well-behaved over the whole region of integration.

For the $\tau$ lepton such an expansion cannot be performed since the integration over hadronic data begins with $\sqrt{s}=2 m_{\pi} \ll m_{\tau}$. However, it is possible to derive an analytic formula for the diagram (2b) which describes the contribution for the $\tau$ with sufficient accuracy.

As a first step, we repeat the calculation of the kernel function for the sum of the 14 diagrams in fig. (2a) using the asymptotic expansions method and confirm the result of [9]. The expansion up to fourth order reads

$$
\begin{aligned}
K^{(2 a)}(s)= & 2 \frac{m^{2}}{s}\left\{\left[\frac{223}{54}-2 \zeta(2)-\frac{23}{36} \ln \frac{s}{m^{2}}\right]\right. \\
& +\frac{m^{2}}{s}\left[\frac{8785}{1152}-\frac{37}{8} \zeta(2)-\frac{367}{216} \ln \frac{s}{m^{2}}+\frac{19}{144} \ln ^{2} \frac{s}{m^{2}}\right] \\
& +\frac{m^{4}}{s^{2}}\left[\frac{13072841}{432000}-\frac{883}{40} \zeta(2)-\frac{10079}{3600} \ln \frac{s}{m^{2}}+\frac{141}{80} \ln ^{2} \frac{s}{m^{2}}\right] \\
& \left.+\frac{m^{6}}{s^{3}}\left[\frac{2034703}{16000}-\frac{3903}{40} \zeta(2)-\frac{6517}{1800} \ln \frac{s}{m^{2}}+\frac{961}{80} \ln ^{2} \frac{s}{m^{2}}\right]\right\} .
\end{aligned}
$$

We now discuss diagram (2b): In the following, $m$ denotes the mass of the external fermion, $m_{f}$ the mass of the fermion in the loop. The result of asymptotic expansions including fifth order is

$$
\begin{aligned}
K^{(2 b)}(s) & =2 \frac{m^{2}}{s}\left\{\left(-\frac{1}{18}+\frac{1}{9} \ln \frac{m^{2}}{m_{f}^{2}}\right)\right. \\
+ & \frac{m^{2}}{s}\left(-\frac{55}{48}+\frac{\pi^{2}}{18}+\frac{5}{9} \ln \frac{s}{m_{f}^{2}}+\frac{5}{36} \ln \frac{m^{2}}{m_{f}^{2}}-\frac{1}{6} \ln ^{2} \frac{s}{m_{f}^{2}}+\frac{1}{6} \ln ^{2} \frac{m^{2}}{m_{f}^{2}}\right) \\
+ & \frac{m^{4}}{s^{2}}\left(-\frac{11299}{1800}+\frac{\pi^{2}}{3}+\frac{10}{3} \ln \frac{s}{m_{f}^{2}}-\frac{1}{10} \ln \frac{m^{2}}{m_{f}^{2}}-\ln ^{2} \frac{s}{m_{f}^{2}}+\ln ^{2} \frac{m^{2}}{m_{f}^{2}}\right) \\
& -\frac{m^{6}}{s^{3}}\left(\frac{6419}{225}-\frac{14}{9} \pi^{2}+\frac{76}{45} \ln \frac{m^{2}}{m_{f}^{2}}-\frac{14}{3} \ln ^{2} \frac{m^{2}}{m_{f}^{2}}-\frac{140}{9} \ln \frac{s}{m_{f}^{2}}+\frac{14}{3} \ln ^{2} \frac{s}{m_{f}^{2}}\right) \\
& \left.-\frac{m^{8}}{s^{4}}\left(\frac{53350}{441}-\frac{20}{3} \pi^{2}+\frac{592}{63} \ln \frac{m^{2}}{m_{f}^{2}}-20 \ln ^{2} \frac{m^{2}}{m_{f}^{2}}-\frac{200}{3} \ln \frac{s}{m_{f}^{2}}+20 \ln ^{2} \frac{s}{m_{f}^{2}}\right)\right\}
\end{aligned}
$$




$$
\begin{aligned}
+2 \frac{m_{f}^{2}}{m^{2}} & {\left[\frac{m^{2}}{s}-\frac{2}{3} \frac{m^{4}}{s^{2}}-\frac{m^{6}}{s^{3}}\left(-2 \ln \frac{s}{m^{2}}+\frac{25}{6}\right)-\frac{m^{8}}{s^{4}}\left(-12 \ln \frac{s}{m^{2}}+\frac{97}{5}\right)\right.} \\
& \left.-\frac{m^{10}}{s^{5}}\left(-56 \ln \frac{s}{m^{2}}+\frac{416}{5}\right)\right] .
\end{aligned}
$$

$K^{(2 a)}$ and $K^{(2 b)}$ can be inserted directly in eq.(6).

For diagrams $(2 \mathrm{~b})$ there are other representations (numerical and analytical) which may be used to check the result from asymptotic expansions.

There are two possible starting points for numerical work:

$$
a^{(2 b)}=\frac{\alpha}{2 \pi^{4}} \int_{4 m_{\pi}^{2}}^{\infty} d s \sigma_{H}(s) \int_{0}^{1} d x \frac{x^{2}(1-x)}{x^{2}+(1-x) \frac{s}{m_{\mu}^{2}}} \cdot \Pi\left(\frac{m_{f}^{2}}{m^{2}}\right),
$$

with either 13

$$
\begin{aligned}
\Pi\left(\frac{m_{f}^{2}}{m^{2}}\right) & =-\frac{8}{9}+\frac{\beta^{2}}{3}-\left(\frac{1}{2}-\frac{\beta^{2}}{6}\right) \beta \ln \frac{\beta-1}{\beta+1} \\
\beta & =\sqrt{1+4 \frac{1-x}{x^{2}} \frac{m_{f}^{2}}{m^{2}}}
\end{aligned}
$$

or [14]

$$
\Pi\left(\frac{m_{f}^{2}}{m^{2}}\right)=2 \int_{0}^{1} d y \cdot y(1-y) \ln \left[1-\left(\frac{-x^{2}}{1-x}\right) \frac{m^{2}}{m_{f}^{2}} y(1-y)\right] .
$$

Finally, we derive an analytical expression for the kernel function, starting from eq.(10). Inspection of eq.(8) shows that, for light fermions in the loop, the terms suppressed with $\frac{m_{f}^{2}}{m^{2}}$ are numerically negligible. We therefore expand $\beta$ in eq.(10) in $\frac{m_{f}^{2}}{m^{2}}$ and retain only unsuppressed terms. After that, the $x$ integration can be performed and we obtain

$$
\begin{aligned}
& K_{\text {analytical }}^{(2 b)}(s)=-\left(\frac{10}{9}+\frac{2}{3} \ln \frac{m_{f}^{2}}{m^{2}}\right) \times \\
& \times\left\{\frac{1}{2}-\left(x_{1}+x_{2}\right)+\frac{1}{x_{1}-x_{2}}\left[x_{1}^{2}\left(x_{1}-1\right) \ln \left(\frac{-x_{1}}{1-x_{1}}\right)-x_{2}^{2}\left(x_{2}-1\right) \ln \left(\frac{-x_{2}}{1-x_{2}}\right)\right]\right\} \\
&-\frac{5}{6}+\frac{2}{3}\left(x_{1}+x_{2}\right)+\frac{2}{3\left(x_{1}-x_{2}\right)}\left\{x_{1}^{2}\left(1-x_{1}\right)\left[\operatorname{Li}_{2}\left(\frac{1}{x_{1}}\right)-\frac{1}{2} \ln ^{2}\left(\frac{-x_{1}}{1-x_{1}}\right)\right]\right. \\
&\left.-x_{2}^{2}\left(1-x_{2}\right)\left[\operatorname{Li}_{2}\left(\frac{1}{x_{2}}\right)-\frac{1}{2} \ln ^{2}\left(\frac{-x_{2}}{1-x_{2}}\right)\right]\right\},
\end{aligned}
$$

with $b=\frac{s}{m^{2}}$ and $x_{1,2}$ and the dilogarithm defined as follows:

$$
\begin{aligned}
x_{1,2} & =\frac{1}{2}\left(b \pm \sqrt{b^{2}-4 b}\right) \\
\operatorname{Li}_{2}(t) & =-\int_{0}^{t} d x \frac{\ln (1-x)}{x} .
\end{aligned}
$$


This expression is exact up to terms of order $\mathcal{O}\left(\frac{m_{f}^{2}}{m^{2}}\right)$. In particular, we still have the full $s$ dependence. Upon expansion of $K_{\text {analytical }}^{(2 b)}(s)$ in $\frac{m^{2}}{s}$ we recover the unsuppressed part of eq.(8).

For the diagram (2c) with two hadronic self energy insertions on the photon propagator the representation is 14

$$
\begin{aligned}
a^{(2 c)} & =\frac{1}{16 \pi^{5} \alpha} \int_{4 m_{\pi}^{2}}^{\infty} d s \sigma_{H}(s) \int_{4 m_{\pi}^{2}}^{\infty} d s^{\prime} \sigma_{H}\left(s^{\prime}\right) \int_{0}^{1} d x \frac{x^{4}(1-x)}{\left[x^{2}+(1-x) \frac{s}{m^{2}}\right]\left[x^{2}+(1-x) \frac{s^{\prime}}{m^{2}}\right]} \\
& =\frac{\alpha^{3}}{9 \pi^{3}} \int_{4 m_{\pi}^{2}}^{\infty} d s d s^{\prime} \frac{R(s) R\left(s^{\prime}\right) K^{(2 c)}\left(s, s^{\prime}\right)}{s \cdot s^{\prime}} .
\end{aligned}
$$

An analytical expression for $K^{(2 c)}\left(s, s^{\prime}\right)$ is easily found; setting $b=\frac{s}{m^{2}}$ and $c=\frac{s^{\prime}}{m^{2}}$ we obtain for $b \neq c$ :

$$
\begin{aligned}
K^{(2 c)}\left(s, s^{\prime}\right)= & \frac{1}{2}-b-c-\frac{(2-b) b^{2} \ln (b)}{2(b-c)}-\frac{b^{2}\left(2-4 b+b^{2}\right) \ln \left(\frac{b+\sqrt{-(4-b) b}}{b-\sqrt{-(4-b) b}}\right)}{2(b-c) \sqrt{-(4-b) b}} \\
& -\frac{(-2+c) c^{2} \ln (c)}{2(b-c)}+\frac{c^{2}\left(2-4 c+c^{2}\right) \ln \left(\frac{c+\sqrt{-(4-c) c}}{c-\sqrt{-(4-c) c}}\right)}{2(b-c) \sqrt{-(4-c) c}},
\end{aligned}
$$

the corresponding formula for $b=c$ is

$$
\begin{aligned}
K^{(2 c)}\left(s, s^{\prime}\right)= & \frac{1}{2}-2 c+\frac{c}{2}(-2+c-4 \ln (c)+3 c \ln (c)) \\
& +\frac{c\left(-2+4 c-c^{2}\right)}{2(-4+c)}+\frac{c\left(12-42 c+22 c^{2}-3 c^{3}\right) \ln \left(\frac{c+\sqrt{(-4+c) c}}{c-\sqrt{(-4+c) c}}\right)}{2(-4+c) \sqrt{(-4+c) c}}
\end{aligned}
$$

\section{Muon}

We take the value for the leading-order hadronic contribution to $a_{\mu}$ from 15] (see [16, 17] for other recent analyses)

$$
a_{\mu}^{(1)}=7023.5(58.5)(140.9) \cdot 10^{-11} .
$$

Here and in the following the first bracket gives the statistical, the second the systematic error. For the higher-order effects we use the combined statistical and systematic error of the leading order as an error estimate.

Higher-order hadronic contributions to the g-2 of the muon were considered for the first time in 1976 [14] and reevaluated in 1984 [18] with new input from experimental data and a new calculation of the light-by-light diagram.

We start with a discussion of the contribution from fig.(2a), employing the expansion eq.(7). If we take only the first line in this formula (this corresponds to eq. $(3.25)$ in [9]) we obtain

$$
a_{\mu}^{(2 a)}(\text { first order })=-200(4) \cdot 10^{-11}
$$


which is very close to the value of $-199 \cdot 10^{-11}$ in 18 . Including the higher-order terms shifts the number to

$$
a_{\mu}^{(2 a)}=-211(5) \cdot 10^{-11} .
$$

We checked the integration over the expanded kernel function against the full formula of ref. [9]. The deviation is below $0.3 \%$ if at least four orders in the expansion are included. It should be noted, however, that the numerical evaluation of eq.(3.21) in [9] for large values of $s$ (i.e. in a region where the expansion converges rapidly) is less reliable than the expansion itself.

We now turn to the contribution from the diagrams of fig. (2b). We restrict ourselves to the case of an electron in the loop. The contribution of the $\tau$ loop is suppressed by $\frac{m_{\mu}^{2}}{m_{\tau}^{2}}$. Using the kernel function of eq.(8) we obtain in agreement with 18

$$
a_{\mu}^{(2 b)}=107(2) \cdot 10^{-11}
$$

Again we notice that at least four or five terms in the expansion must be included in order to obtain a stable result. The number decreases from $126 \cdot 10^{-11}$ in first order to the value of eq.(19) in fifth order (between 4 th and 5 th order the value changes by $0.2 \%$ ). The part of eq.(8) suppressed with $\frac{m_{e}^{2}}{m_{\mu}^{2}}$ turns out to be numerically completely negligible.

The result of eq.(19) has been checked using different numerical integration methods and the analytical representation of eq.(12). Whereas the analytical result coincides with the expansion, care has to be taken in the numerical integrations.

Numerically, the representation eq.(10) is much less appropriate than eq.(11). The latter is a flat function and the double numerical integration over $x$ and $y$ yields the result in eq.(19). The representation eq.(10) is a peaked function and although for fixed $s$ the error is at worst of the order of $1 \%$ it adds up systematically in the $s$ integration and reaches $10 \%$ in the final result.

To conclude the discussion for the muon, we give the value we obtain for diagram (2c) with two hadronic bubbles on the photon propagator. Using eq.(13) we obtain

$$
a_{\mu}^{(2 c)}=2.7(0.1) \cdot 10^{-11}
$$

This number can be reproduced using the analytical expressions eq.(14,15) with quadruple precision Fortran.

We find a total shift in the higher-order hadronic contributions of $a_{\mu}$ from [18]

$$
a_{\mu}^{(2 a+2 b+2 c)}=-90(5) \cdot 10^{-11}
$$

to

$$
a_{\mu}^{(2 a+2 b+2 c)}=-101(6) \cdot 10^{-11} .
$$

The difference $(-11) \cdot 10^{-11}$ is somewhat smaller but still of the same order of magnitude as the experimental uncertainty in the BNL experiment, or approximately $25 \%$ 
of the two-loop electroweak contributions. It should be emphasized that the reason for the shift doesn't reside predominantly in the input of new experimental data; rather, the high precision of the Brookhaven experiment requires the use of the full kernel functions.

\section{Electron}

Higher-order hadronic contributions to the g-2 of the electron have only recently [2] been considered for the first time. Some features are special to the electron (as opposed to the muon case) and will be discussed in the following.

For the electron the calculation of diagram (2a) is very similar to the muon case. It is sufficient to take the first term in the expansion of eq.(77).

$$
K^{(2 a)}(s)=-2 \frac{m_{e}^{2}}{s}\left(\frac{23}{36} \ln \frac{s}{m_{e}^{2}}+\frac{\pi^{2}}{3}-\frac{223}{54}\right) .
$$

Here, the contribution from the large logarithm dominates in the whole region of integration. We obtain the value

$$
a_{e}^{(2 a)}=-2.25(0.05) \cdot 10^{-13}
$$

The diagrams of fig. (2b) with an external electron yield a numerically negligible contribution. The first observation is that the diagrams with an electron self-energy on the photon propagator are already contained in the set of diagrams (2a). Diagrams with a muon or a $\tau$ lepton on the photon propagator are suppressed by additional powers of $\frac{m_{e}^{2}}{m_{\mu}^{2}}$ or $\frac{m_{e}^{2}}{m_{\tau}^{2}}$ respectively, and hence can be omitted. This is a special case of the general statement that in diagrams of the type (2b) large logarithms occur if the mass of the external fermion is bigger than the mass of the fermion in the loop (see, for instance, the leading term $\frac{1}{9} \ln \frac{m_{\mu}^{2}}{m_{e}^{2}}$ in eq.(8)); in the opposite case there is a mass suppression. The double hadronic bubble diagram (2c) is of the order $a_{e}^{(2 c)} \approx 1.2 \cdot 10^{-19}$ for the same reason.

For an estimate of the total higher-order hadronic contribution to $a_{e}$ including lightby-light scattering we refer to ref. [2]:

$$
a_{e}^{(2 a)}+a_{e}^{(3)}=(-2.25-0.122) \cdot 10^{-13}=-0.24 \cdot 10^{-12},
$$

where the light-by-light value has been estimated by rescaling the corresponding number for the muon.

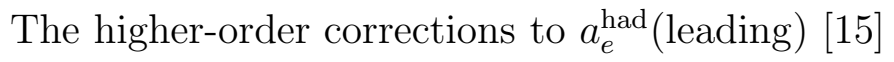

$$
a_{e}^{(1)}=1.8847(0.0165)(0.0375) \cdot 10^{-12}
$$

reduce the total hadronic contribution by $13 \%$; the final number is

$$
a_{e}^{\text {had }}(\text { total })=1.645 \cdot 10^{-12} .
$$




\section{$\tau$ lepton}

The leading-order hadronic effect is [15]

$$
a_{\tau}^{(1)}=338.30(1.97)(9.12) \cdot 10^{-8}
$$

In [19 an estimate of the higher-order hadronic contributions to $a_{\tau}$ was given

$$
a_{\tau}^{\text {had }}(\text { higher orders })=-1.2(2) \cdot 10^{-7}
$$

The number can be reproduced by rescaling the muon results of [18] with the factor $\frac{m_{\tau}^{2}}{m_{\mu}^{2}}$ and taking the 1991 value for $m_{\tau}$ :

$$
\frac{m_{\tau}^{2}}{m_{\mu}^{2}}\left(a_{\tau}^{(2 a+2 b+2 c)}+a_{\tau}^{l i g h t-b y-l i g h t}\right)=(-90+49) \cdot 10^{-11} \cdot\left(\frac{1784}{106}\right)^{2}=-1.2 \cdot 10^{-7} .
$$

It will be shown in the following that, as in the case of the electron, simple rescaling of the muon results doesn't give a correct estimate.

For the $\tau$ lepton it is no longer possible to use expansions in $\frac{m_{\tau}^{2}}{s}$ over the whole region of integration because the integration starts with $s=4 m_{\pi}^{2}$. The diagrams of fig. (2a) can still be calculated with the general kernel function of [9]. We obtain the value

$$
a_{\tau}^{(2 a)}=-3.18(0.08) \cdot 10^{-8} .
$$

In the case of the diagrams of fig. (2b) we have to sum over the contributions of the electron and the muon in the loop. Here, we apply two different methods to compute the anomaly: a two-dimensional numerical integration yields the results

$$
\begin{aligned}
a_{\tau}^{(2 b)}(\text { electron }) & =7.48(0.2) \cdot 10^{-8} \\
a_{\tau}^{(2 b)}(\text { muon }) & =1.91(0.05) \cdot 10^{-8} .
\end{aligned}
$$

Using the analytic formula eq.(12) we obtain the following values:

$$
\begin{aligned}
a_{\tau}^{(2 b)}(\text { electron }) & =7.48(0.2) \cdot 10^{-8} \\
a_{\tau}^{(2 b)}(\text { muon }) & =1.88(0.05) \cdot 10^{-8} .
\end{aligned}
$$

As expected, the electron contribution is the same and we have a $2 \%$ deviation for the muon. This is consistent with the order of magnitude of the neglected terms, i.e.

$$
\frac{m_{\mu}^{2}}{m_{\tau}^{2}} \ln \frac{m_{\tau}^{2}}{m_{\mu}^{2}} \approx 0.02
$$

For the $\tau$ lepton, diagram (2c) also yields a numerically important contribution. From eq.(13) we get the value

$$
a_{\tau}^{(2 c)}=1.4(0.1) \cdot 10^{-8}
$$

The higher-order correction to $a_{\tau}^{\text {had }}$ (leading) enhances the total hadronic contribution by $2 \%$ (without the light-by light contribution):

$$
a_{\tau}^{(2 a+2 b+2 c)}=7.6(0.2) \cdot 10^{-8} .
$$


This should be contrasted with the estimate from simple rescaling:

$$
\left(\frac{m_{\tau}^{2}}{m_{\mu}^{2}}\right) a_{\mu}^{(2 a+2 b+2 c)}=(-101) \cdot 10^{-11} \cdot\left(\frac{1777}{106}\right)^{2}=-2.8 \cdot 10^{-7} .
$$

Keeping this uncertainty in mind, we give an estimate for higher-order hadronic contributions to $a_{\tau}$ with eq.(37) and a rescaled light-by-light value from [6]:

$$
a_{\tau}^{\text {had }}(\text { higher orders }) \approx\left(\frac{m_{\tau}^{2}}{m_{\mu}^{2}}\right) \cdot\left(-52 \cdot 10^{-11}\right)+7.6 \cdot 10^{-8}=-7.0 \cdot 10^{-8} .
$$

\section{Conclusions}

We presented analytic formulae for the calculation of higher-order hadronic contributions to the g-2 of leptons and introduced the method of asymptotic expansions as a convenient tool to calculate the corresponding kernel functions. We demonstrated that in order to match the high experimental precision of the upcoming experiments, it is necessary to include the full kernel functions in theoretical predictions. The higher-order hadronic effects on the anomalous magnetic moments of the muon, electron, and $\tau$ lepton are presented. The remaining uncertainties in the predictions from the Standard Model reside now in the experimental error in the measurement of $\sigma\left(e^{+} e^{-} \rightarrow\right.$ hadrons $)$ and the calculation of light-by-light-scattering effects.

\section{Acknowledgements}

I would like to thank F. Jegerlehner and S. Eidelman for the permission to use their set of experimental data. I am grateful to A. Czarnecki, T. Kinoshita, J. H. Kühn and T. Teubner for useful discussions and advice, and M. L. Stong for reading of the manuscript. I would like to thank A. Höcker for checking part of the numerical results and useful comments. This work was supported by "Graduiertenkolleg Elementarteilchenphysik" at the University of Karlsruhe.

\section{References}

[1] V. W. Hughes, in Frontiers of High Energy Spin Physics, edited by T. Hasegawa et al. (Universal Academy Press, Tokyo, 1992), pp. 717-722.

[2] T. Kinoshita, Cornell preprint CLNS-96-1406 (1996).

[3] M. Perl, Nucl. Phys. B40 (Proc. Suppl.), 541 (1995).

[4] W. Marciano, Nucl. Phys. B40 (Proc. Suppl.), 3 (1995).

[5] S. Eidelman and F. Jegerlehner, Z. Phys. C67, 585 (1995).

[6] M. Hayakawa, T. Kinoshita, and A. I. Sanda, Phys. Rev. Lett. 75, 790 (1995), and M. Hayakawa, T. Kinoshita, and A. I. Sanda, DPNU-95-30, hep-ph/9601310. 
[7] J. Bijnens, E. Pallante, J. Prades, Phys. Rev. Lett. 75, 1447, (1995); Erratum: ibid. 75, 3781 (1995), and J. Bijnens, E. Pallante, J. Prades, preprint NORDITA95-75-N-P, hep-ph/9511388.

[8] S.J. Brodsky, E. de Rafael, Phys. Rev. 168, 1620 (1968).

[9] R. Barbieri, E. Remiddi, Nucl. Phys. B90, 233 (1975).

[10] For a recent review see V. A. Smirnov, Mod. Phys. Lett. A10, 1485 (1995).

[11] A. Czarnecki, B. Krause, and W. Marciano, Phys. Rev. D52, R2619 (1995).

[12] A. Czarnecki, B. Krause, and W. Marciano, Phys. Rev. Lett. 76, 3267 (1996).

[13] M. Samuel and G. Li, Phys. Rev. D44, 3935 (1991); Erratum D48, 1879 (1993).

[14] J. Calmet, S. Narison, M. Perrottet and E. de Rafael, Phys. Lett. B61, 283 (1976).

[15] F. Jegerlehner, in Proceedings of the Zeuthen Workshop on Elementary Particle Theory: QCD and QED in Higher Orders, Rheinsberg, Germany, 21-26 Apr. 1996, preprint DESY-96-121.

[16] K. Adel and F. Ynduráin, FTUAM 95-2 (1995).

[17] W. Worstell and D. Brown, Boston University preprint (1995).

[18] T. Kinoshita, B. Nizic, and Y. Okamoto, Phys. Rev. D31, 2108 (1985).

[19] G. Li, R. Mendel and M. Samuel, Phys. Rev. Lett. 67, 668 (1991); 


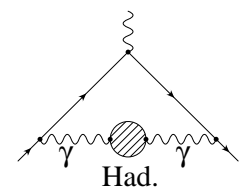

Fig. (1): Leading order hadronic contribution
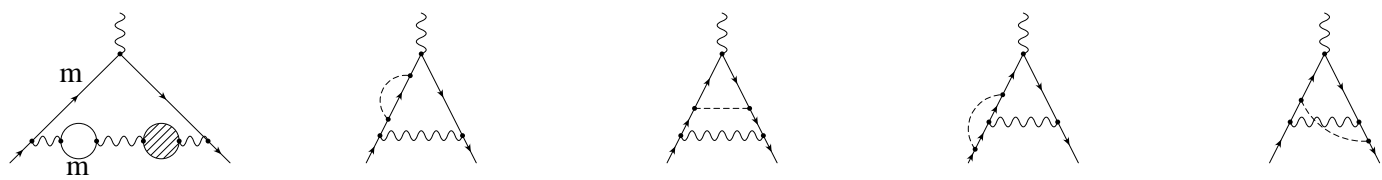

Fig. (2a): Dashed lines indicate a hadronic insertion on the photon propagator. Mirror counterparts and diagrams with interchange of massless and "massive" photon propagators have to be included.

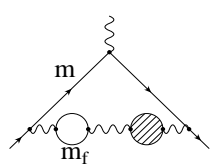

Fig. (2b)

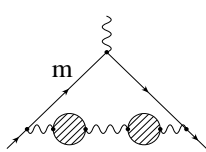

Fig. (2c)

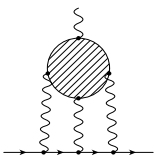

Fig. (3) 


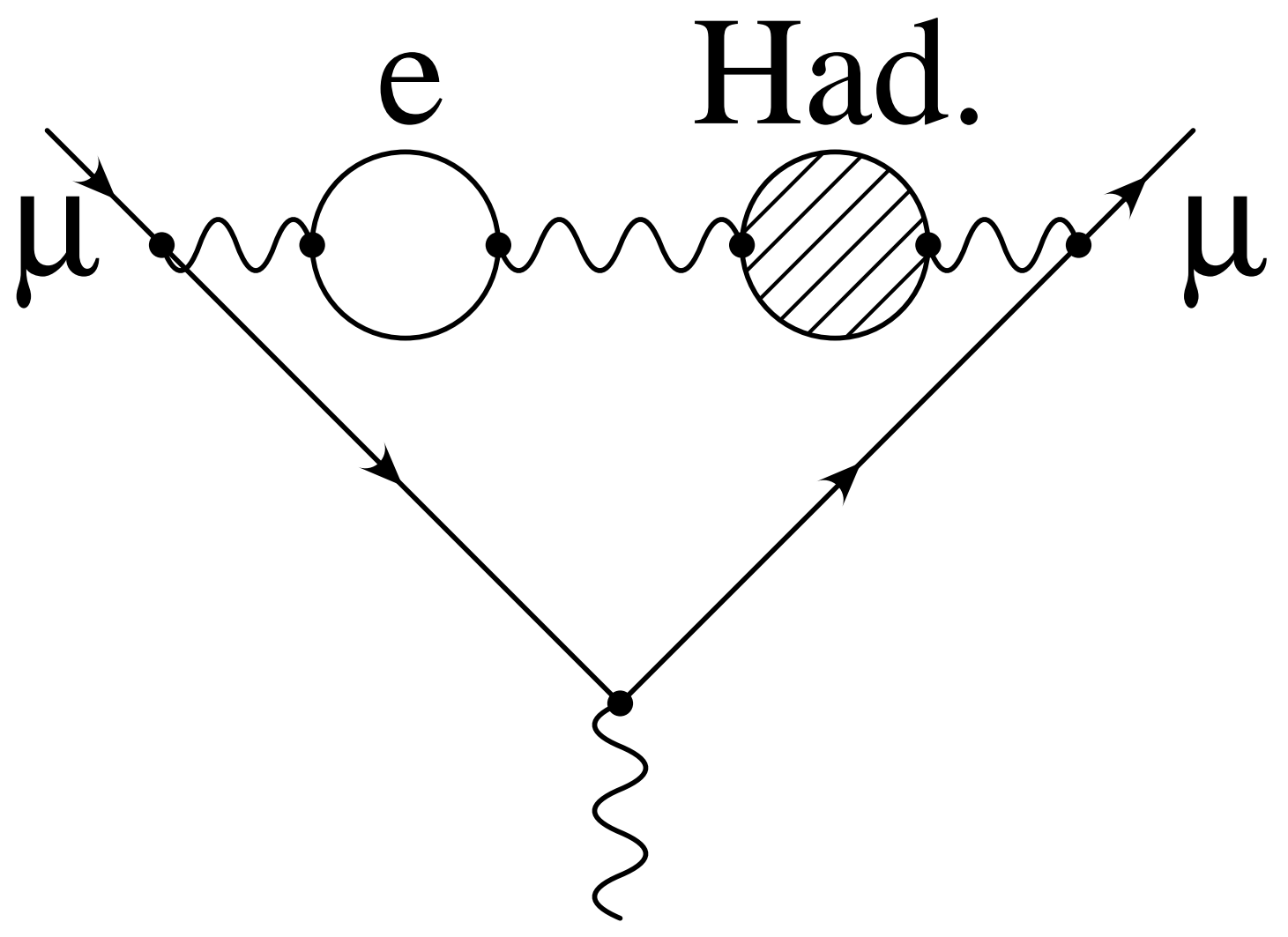

